\title{
Effect of variation in herkogamy on outcrossing within a population of Gilia achilleifolia
}

\author{
N Takebayashi, DE Wolf ${ }^{1}$ and LF Delph \\ Department of Biology, Indiana University, Bloomington, Indiana 47405-3700, USA
}

\begin{abstract}
The diverse features of floral morphology are often thought to be well-designed mechanisms to manipulate plant mating systems. We evaluated the effectiveness of one such mechanism, anther-stigma separation (herkogamy), in controlling variation in the level of outcrossing among plants in a population of Gilia achilleifolia. Variation in outcrossing rates within populations has the potential to influence the coevolution between inbreeding depression and mating system. Using four polymorphic allozymes, we compared the outcrossing-rate estimates of two groups of individuals under natural conditions: one group with low herkogamy and another with high herkogamy. The high herkogamy group had a higher outcrossing rate $(0.572)$ than the low herkogamy group (0.335). This suggests that the withinpopulation variation in outcrossing rate could potentially cause the previously observed association between herkogamy and inbreeding depression (Takebayashi and
\end{abstract}

Delph, 2000). A previous study of floral traits among G. achilleifolia populations failed to detect a relationship between herkogamy and outcrossing rate, demonstrating that the functionality of traits may be obscured in amongpopulation studies as a consequence of uncontrolled environmental variation. Additionally, the effect of herkogamy on outcrossing rate in delayed selfers such as $G$. achilleifolia may be particularly prominent when pollinator availability is low. Our population-level estimate of outcrossing rate (0.444) was somewhat lower than an estimate from the same population, 15 years prior to our study (0.75), suggesting that pollinator availability may fluctuate among years. Both within-year and among-year variation in the outcrossing rate may have a strong influence on matingsystem evolution.

Heredity (2006) 96, 159-165. doi:10.1038/sj.hdy.6800780; published online 21 December 2005

Keywords: autogamy; herkogamy; floral design; variation in outcrossing rate; plant mating systems; temporal variation

\section{Introduction}

Plant mating-system evolution is a dynamic process, as evidenced by the wide variation seen in outcrossing rates among closely related species and among populations within species (eg Grant and Grant, 1965; Barrett and Eckert, 1990; Takebayashi and Morrell, 2001). An understanding of the forces governing plant mating-system evolution is fundamental to the understanding of evolution in plant populations, because mating system influences evolutionary factors as diverse as the amount and pattern of genetic variation within and among populations (Loveless and Hamrick, 1984), reproductive assurance (Sakai and Ishii, 1999), ability to colonize new populations (Baker, 1955), effective recombination rate (Wright et al, 2002), and long-term evolvability (Takebayashi and Morrell, 2001).

Various aspects of floral design are frequently invoked as adaptations, either for the promotion or avoidance of self-fertilization (Darwin, 1876). For example, the temporal and spatial separation of female and male functions within a flower (dichogamy and herkogamy, respectively) are expected to enhance outcrossing by

Correspondence: N Takebayashi. Current address: 311 Irving 1, Institute of Arctic Biology and Department of Biology and Wildlife, University of Alaska Fairbanks, Fairbanks, AK 99775, USA. E-mail: ffnt@uaf.edu

${ }^{1}$ Current address: 311 Irving 1, Institute of Arctic Biology and Department of Biology and Wildlife, University of Alaska Fairbanks, Fairbanks, AK 99775, USA

Received 10 May 2005; accepted 7 November 2005; published online 21 December 2005 reducing the interference between the two functions (Lloyd and Webb, 1986; Webb and Lloyd, 1986). Conversely, in autonomously selfing species, natural selection may minimize the degree of herkogamy such that the filament and stigma heights will be tightly correlated (Ushimaru and Nakata, 2002). However, outcrossing rate is a complex character that can be influenced by many factors, such as total floral display, local plant density, pollinator behavior, and pollinator abundance (Kunin, 1993; Karron et al, 1995; Vrieling et al, 1999). These extrinsic factors may create sufficient variation that floral morphology has little influence on the realized outcrossing rate in natural populations.

Most attempts to determine the effect of floral traits on outcrossing have made comparisons among closely related species or among conspecific populations (eg Schoen, 1982a; Holtsford and Ellstrand, 1992). Such studies have shown among-population correlations between the average amount of herkogamy and outcrossing rates (Rick et al, 1977; Holtsford and Ellstrand, 1992; Belaoussoff and Shore, 1995). Less known is the extent of within-population variation in outcrossing rates and the factors influencing such variation, even though this is one of the primary levels at which natural selection acts. Such mating-system variation among individuals within populations may have an important influence on the coevolution between outcrossing rate and inbreeding depression (see below).

Inbreeding depression is one of the primary forces influencing whether outcrossing or self-fertilization 
should be favored (Kimura, 1959). Self-fertilization increases homozygosity, which can reveal partially recessive deleterious mutations, causing reduction in fitness of selfed offspring. When inbreeding depression is strong (the fitness of selfed offspring is less than half that of outcrossed offspring), outcrossing is generally thought to be favored (Kimura, 1959). Conversely, outcrossing rate also influences the magnitude of inbreeding depression. When deleterious recessive mutations are revealed by self-fertilization, they are eliminated by selection. Thus, inbreeding depression is expected to be reduced by self-fertilization, and it can accumulate in outcrossing populations (Lande and Schemske, 1985). Classically, inbreeding depression has been treated as a characteristic of a population. However, when outcrossing rates differ among individuals within populations, individuals vary in inbreeding history. Variation in inbreeding history may potentially result in lineage-specific purging of genetic load and consequently different magnitudes of inbreeding depression among lineages within populations. Through this process, genetic associations may develop between loci controlling outcrossing rate and viability loci that cause inbreeding depression (Uyenoyama and Waller, 1991). Theoretically, such genetic associations can alter the balance between the costs and benefits of outcrossing, influencing the spread of morphological or genetic traits that control the outcrossing rate.

Despite the significant influence of genetic associations between loci controlling viability and outcrossing rate on the outcomes of mating-system evolution, it is not yet clear how common these associations are, and whether they really arise by the lineage-specific purging process. A previous study of Gilia achilleifolia appears to demonstrate that such associations exist; highly herkogamous individuals showed more severe inbreeding depression than individuals with low anther-stigma separation (Takebayashi and Delph, 2000). Several other studies have also found similar correlations between family-level inbreeding depression and traits that influence outcrossing rate (Vogler et al, 1999; Stone and Motten, 2002). However, a multilocus simulation study suggested that genetic associations are unlikely to be observed (Schultz and Willis, 1995). Further, several empirical studies failed to detect any such association (Carr et al, 1997; Mutikainen and Delph, 1998; Rao et al, 2002). In order to understand the association observed in $G$. achilleifolia, it is of particular importance to assess how effectively herkogamy can control outcrossing rate within a natural population. The association will not develop by the proposed mechanism if variation in the outcrossing rate is controlled more by environmental variation than by floral morphology. Here, we examine the effect of herkogamy on outcrossing rates under natural conditions in the wild.

\section{Materials and methods}

Recently, researchers have begun to investigate the effects of floral variation on individual outcrossing rates within a population (Barrett et al, 1994; Brunet and Eckert, 1998; Vrieling et al, 1999; Motten and Stone, 2000). Current procedures for estimating population-level outcrossing rates are quite robust if multiple loci are included in the study (Ritland and Jain, 1981). However, the reliability is reduced when analogous methods, based on maximum likelihood (Ritland and Jain, 1981) or an extension of method-of-moments procedure (Cruzan et al, 1994), are used to estimate outcrossing rates of individuals within a population (Ritland and Ganders, 1985; Morgan and Barrett, 1990; Ivey and Wyatt, 1999). This is in part due to unequal detection of outcrossing events in different maternal genotypes (Morgan and Barrett, 1990). For instance, while outcrossing events can be reliably detected in maternal plants that are homozygous for rare alleles, estimates for maternal plants with common genotypes depend on pollen allele frequencies and are therefore less reliable. One way to avoid the problem is to use an artificial plot in which the genotypic frequencies are controlled (eg Motten and Stone, 2000). Alternatively, one can compare the outcrossing-rate estimates of two groups differing in some focal trait (Brunet and Eckert, 1998). We adopted the latter approach for our study.

G. achilleifolia is endemic to the central Coast Range of California. The species is self-compatible with hermaphroditic flowers and is pollinated by insects, including honeybees, halictid and andrenid bees, and beeflies (Grant and Grant, 1965). Different populations are reported to have widely divergent outcrossing rates (Schoen, 1982a), as well as wide variation in traits that may influence outcrossing rates, such as flower size, degree of protandry (amount of time separating anther dehiscence and stigma receptivity), and degree of herkogamy (physical separation between anthers and stigma) (Schoen, 1982a).

The study was conducted in the Arroyo Seco population, Monterey County, California, a mainly outcrossing population, with an estimated outcrossing rate of 0.75 (Schoen, 1982a). From this population of more than 1000 plants, we haphazardly selected 38 target plants, in which we measured herkogamy and outcrossing. These plants were tagged for latter identification.

Five flowers, each from a different inflorescence, were collected from each plant between 16 and 20 May 1995, and stored in $70 \%$ ethanol. The flowers were collected at or slightly after the peak of the flowering season. The anther-stigma separation of each flower was estimated under a dissecting microscope, as the height of the lowest stigma lobe minus the height of the highest anther (details in Takebayashi and Delph, 2000).

Open-pollinated fruits were collected from the 38 target plants for isozyme analysis on June 1 and 12, 1995. Since fruits mature about 3 weeks after pollination (pers. obs.), the first fruits collected (June 1) were from flowers pollinated slightly before the peak of flowering, and the fruits collected on June 12 correspond to flowers pollinated during the flower collection, near the peak of flowering. Of the 38 plants in this study, seven were excluded from analysis; three plants failed to produce a sufficient number of fruits for isozyme analysis, and four plants produced primarily nonfunctional anthers, such that anther-stigma distance would clearly not influence outcrossing rates in these individuals.

Two groups of individuals were selected for the isozyme analysis, based on the estimates of antherstigma separation. Out of 31 plants (mean $=-0.26 \mathrm{~mm}$, standard deviation $=0.65$ ), we chose approximately $1 / 3$ at each end of the distribution, the 12 individuals with the smallest separation (mean $=-0.89 \mathrm{~mm}$, standard 
deviation $=0.50)$ and the 12 with the largest (mean $=0.34 \mathrm{~mm}$, standard deviation $=0.30$ ) were used. Anther-stigma distances were unimodally distributed, so the cutoff between the two groups was somewhat arbitrary. This sample size (1/3) was chosen prior to beginning the study by considering the compromise between the sampling efforts and detection of the effect of herkogamy on outcrossing rates. Isozyme analysis was conducted on 20-26 seeds per family (mean $=20.8$ ), and seeds were sampled such that no more than two seeds came from a single fruit.

In an initial isozyme screening of nine enzymes, four were found to be polymorphic: alcohol dehydrogenase $(A D H$, E.C.1.1.1.1), glucose phosphate isomerase (GPI, E.C.5.3.1.9), isocitric acid dehydrogenase (IDH, E.C.1.1.1.42), and phosphoglucomutase (PGM, E.C.2.7.5.1). The other five enzymes were monomorphic: malate dehydrogenase (E.C.1.1.1.37), malic enzyme (E.C.1.1.1.40), 6-phosphogluconate dehydrogenase (E.C.1.1.1.44), glucose dehydrogenase (E.C.1.1.1.47), and mannose-phosphate isomerase (E.C.5.3.1.8).

Multi-locus genotypes of each seed were obtained using the four polymorphic enzymes, by the following procedure. The mucilaginous seed coat, which causes blurry isozyme bands, was removed after soaking in water for $1 \mathrm{~h}$. Each seed was then homogenized in $12 \mu \mathrm{l}$ of extraction buffer (0.1 M Tris- $\mathrm{HCl}$ pH8.0, $0.147 \mathrm{M}$ sucrose, $0.24(\mathrm{v} / \mathrm{v}) \%$ Triton $\mathrm{X}, 5(\mathrm{v} / \mathrm{v}) \% \beta$-mercaptoethanol), and centrifuged at 8000 r.p.m for $6 \mathrm{~min}$ to remove cell debris. Using the Helena Research Laboratory Zip Zone system (Hebert and Beaton, 1989), cellulose acetate gels (Gelman Sciences, Optiphor-10) were run for $1 \mathrm{~h}$ at $140 \mathrm{~V}$ for all enzymes except ADH, which was run at $120 \mathrm{~V}$. Three electrophoretic buffer systems of Richardson et al (1986) were used for both soaking and running the gels (ie continuous buffer system). GPI was resolved with Buffer System D (15 mM Tris, $5 \mathrm{mM}$ EDTA, $10 \mathrm{mM} \mathrm{MgCl}_{2}, 5.5 \mathrm{mM}$ boric acid, pH7.8), while Buffer System F (100 mM Tris, 1mM EDTA, $1 \mathrm{mM} \mathrm{MgCl} 2,43.1 \mathrm{mM}$ maleic acid, $\mathrm{pH7.4)}$ was used for PGM and ADH. For IDH, Tris-citrate buffer $(100 \mathrm{mM}$ Tris, $34.5 \mathrm{mM}$ citric acid, pH6.5) was employed. Staining protocols were as described in Richardson et al (1986) except that the $\mathrm{pH}$ of $0.1 \mathrm{M}$ Tris- $\mathrm{HCl}$ was changed to 7.5 in the $\mathrm{ADH}$ stain, and gels were stained at $39^{\circ} \mathrm{C}$ to intensify enzymatic reactions.

The maximum-likelihood based program, MLTR (version 3.0; Ritland, 2002) was used to estimate parental inbreeding coefficients $(F)$, ovule and pollen allele frequencies, and mating-system parameters. Matingsystem parameters that were estimated include: singleand multi-locus outcrossing rates $\left(t_{m}\right.$ and $t_{s}$, respectively; Ritland and Jain, 1981) and correlation of paternal parentage through self-fertilization and through outcrossing between sibs ( $r_{s}$ and $r_{p}$, respectively; Ritland, $1988,2002)$, and correlation of selfing rates among loci $\left(r_{s, l o c i} ;\right.$ Ritland, 2002). The traditional mixed-mating model (eg Ritland and Jain, 1981) assumes that a fraction of progeny are produced through self-fertilization and the remaining progeny are derived from random outcrossing. The correlated-matings model, used in MLTR, incorporates an additional aspect of plant mating systems: that siblings may share the same male parent. For instance, if a plant is sired only by the closest neighbor, paternal parentage among the outcrossed sibs is highly correlated (ie high $r_{p}$ ). Under low pollinator availability, a high correlation of selfing $\left(r_{s}\right)$ is also likely because seeds derived from a flower that has been visited may all be outcrossed, whereas seeds derived from an un-visited flower will all be selfed. Biparental inbreeding can be suggested by low $r_{s, l o c i}$ because biparental inbreeding causes variation in $t_{s}$ and reduces the correlation of $t_{s}$ among loci (Ritland, 2002). Maximum-likelihood estimates of mating-system parameters were calculated for the two groups of individuals by assuming that both groups experienced the same pollen allele frequencies. Maternal genotypes were inferred from the progeny arrays by the maximum-likelihood method of Brown and Allard (1970). Newton-Raphson iteration was used to find the maximum-likelihood estimates for all parameters. The standard error of each estimate was calculated as the standard deviation of 1000 bootstrap values with the maternal progeny array as the unit of resampling.

\section{Results}

A one-way ANOVA detected substantial phenotypic variation in herkogamy among plants $\left(F_{33,136}=4.44\right.$, $\left.P<10^{-9}\right)$. The amount of variation was comparable to that in the previous study (Takebayashi and Delph, 2000) where herkogamy was measured in greenhouse-grown plants from the same population (Bartlett's $K^{2}=2.39$, $d f=1, P=0.12$ ). The population mean for herkogamy $(-0.28 \mathrm{~mm})$ is smaller than in the previous greenhouse study $(0.49 \mathrm{~mm} ; t=-4.36, d f=66.9, P<0.0001)$. In the previous study, we measured herkogamy from fresh flowers, so the difference may be caused by some shrinkage of flowers in $70 \%$ ethanol.

We observed two ADH loci, but only the fastmigrating locus (Adh-1) was polymorphic (Table 1), having five alleles, rather than the two alleles observed previously in the same population (Schoen, 1982b). This difference is probably due to increased resolution on cellulose acetate gels compared to the starch gels used by Schoen (1982b). Of the two loci found with GPI, only the slow-migrating locus (Gpi-2) was polymorphic, having three alleles. Frequencies of the Gpi-2 alleles (Table 1) were comparable to those in the previous study: 0.18 for the fastest allele, 0.77 for the middle allele, and 0.05 for the slowest (Schoen, 1982b). The Idh locus and the fast Pgm-1 locus showed similar patterns; one of the two alleles at each locus was very rare, with a frequency less than 0.06. The slow Pgm-2 locus was not used because the weak expression of the enzyme precluded consistent scoring of all electromorphs. Across all loci, the parametric estimate of Wright's fixation index $(F)$ in the parents was $0.140(\mathrm{SE}=0.142)$.

Our primary objective was to examine the prediction that the level of outcrossing should increase with herkogamy. As expected, individuals with high herkogamy showed significantly higher multi-locus and single-locus estimates of outcrossing rates than those with low herkogamy (Table 2). The difference between the two groups was surprisingly large (about 24\%). Additionally, both high and low herkogamy groups showed a high correlation of selfing $\left(r_{s}\right)$ (Table 2$)$. The high herkogamy group showed a high correlation of outcrossed paternity $\left(r_{p}\right)$ within maternal family (Table 2) while $r_{p}$ was not significantly different from 0 in the low 
Table 1 Allele frequencies of maternal parents and pollen pools in the Arroyo Seco population of G. achilleifolia

\begin{tabular}{lccc}
\hline Locus & Allele & Pollen & Ovule \\
\hline Adh-1 & $\mathrm{a}$ & $0.136(0.052)$ & $0.063(0.046)$ \\
& $\mathrm{b}$ & $0.415(0.077)$ & $0.375(0.078)$ \\
& $\mathrm{c}$ & $0.174(0.030)$ & $0.333(0.075)$ \\
& $\mathrm{d}$ & $0.245(0.068)$ & $0.167(0.057)$ \\
& $\mathrm{e}$ & $0.031(0.016)$ & $0.063(0.035)$ \\
Adh-2 & & Monomorphic & \\
Gpi-1 & & Monomorphic & \\
Gpi-2 & & $0.156(0.040)$ & $0.042(0.028)$ \\
& $\mathrm{a}$ & $0.755(0.051)$ & $0.792(0.067)$ \\
& $\mathrm{b}$ & $0.089(0.034)$ & $0.167(0.057)$ \\
Idh & $\mathrm{c}$ & $0.943(0.021)$ & $0.958(0.028)$ \\
& & $0.057(0.021)$ & $0.042(0.028)$ \\
Pgm-1 & $\mathrm{b}$ & $0.023(0.014)$ & $0.021(0.020)$ \\
& & $0.977(0.014)$ & $0.979(0.020)$ \\
\hline
\end{tabular}

Locus ' 1 ' represents the fastest, anodally-migrating locus for a system. Allele ' $a$ ' represents the fastest, anodally-migrating allele. The standard errors (in parentheses) were estimated by 1000 bootstraps of the data.

Table 2 Mating system parameters estimated from natural populations of G. achilleifolia

\begin{tabular}{lrrr}
\hline Parameter & \multicolumn{2}{c}{ Group mean (SE) } & Difference (SE) \\
\cline { 2 - 3 } & Low herkogamy & High herkogamy & \\
\hline$t_{m}$ & $0.335(0.063)$ & $0.572(0.063)$ & $0.238(0.085)$ \\
$t_{s}$ & $0.331(0.064)$ & $0.493(0.051)$ & $0.162(0.077)$ \\
$r_{s}$ & $0.362(0.184)$ & $0.467(0.209)$ & $0.105(0.271)$ \\
$r_{p}$ & $-0.042(0.146)$ & $0.408(0.255)$ & $0.449(0.286)$ \\
$r_{s, \text { loci }}$ & $0.800(0.192)$ & $0.035(0.272)$ & $-0.765(0.318)$ \\
\hline
\end{tabular}

The estimates of high and low herkogamy groups and the difference between the two groups are shown. Estimates of the standard error (in parentheses) were obtained from 1000 bootstraps of the data. $t_{m}=$ multi-locus outcrossing rate, $t_{s}=$ mean single-locus outcrossing rate, $r_{s}=$ correlation of selfing, $r_{p}=$ multi-locus correlation of outcrossed paternity within progeny array, $r_{s, l o c i}=$ correlation of selfing among loci.

herkogamy group. In the high herkogamy group, the correlation of selfing among loci $\left(r_{s, l o c i}\right)$ was surprisingly low, suggesting that they experienced strong biparental inbreeding. This was not observed in the low herkogamy group.

\section{Discussion}

\section{Allelic diversity}

Allelic diversity was surprisingly high at the ADH locus (five electromorphs). Isozyme analysis from several populations of G. achilleifolia showed that most of other polymorphic enzymes were diallelic, with the occasional triallelic loci (Takebayashi and Morrell, unpublished data). In several diverse species, studies of DNA sequence variation indicate that $\mathrm{ADH}$ loci are potentially subject to balancing selection (Kreitman and Hudson, 1991; Savolainen et al, 2000; Miyashita, 2001). High ADH diversity in G. achilleifolia may also be maintained by some form of stabilizing selection. Since the neutrality of genetic markers is assumed in models of mating-system estimation, our estimates could potentially be biased. However, because we isolated isozymes from seeds rather than from germinated seedlings, the bias should be minimal, as long as Adh-1 genotypes do not influence the fitness in early life-stages such as pollen tube growth, ovule fertilization, or seed maturation.

\section{Characterization of mating system}

We found a positive correlation of selfing within progeny arrays (Table 2). Correlation of selfing $\left(r_{s}\right)$ can be interpreted as the normalized variance of the selfing rate among families (Ritland, 2002), or as a measure of departure from an assumption in the 'mixed-mating' model, which is commonly used for estimating outcrossing rates (Ritland and Jain, 1981). The mixed-mating model assumes that the probability of a particular mating event (selfing or outcrossing) is statistically independent of other mating events in the same progeny array. If this assumption is satisfied, $r_{s}$ estimates are close to zero. Generally, correlated selfing arises when plants vary in floral traits or pollinator visitation rates, and hence in outcrossing rates. A positive value of $r_{s}$ means that two selfed progeny sampled from the same progeny array are not the result of two independent selfing events. For instance, such correlated selfing may occur when movement of a pollinator causes within-flower selfing. Therefore such correlations are expected to be high among seeds within a single fruit. Although we tried to sample seeds from as many fruits as possible, we had to use several fruits from the same inflorescence. Because G. achilleifolia inflorescences are often densely packed with 2-30 flowers, correlated selfing may be high not just among seeds within a fruit but also among fruits within an inflorescence. Similarly, mating events are not expected to be statistically independent in a delayed selfer, such as G. achilleifolia; outcrossing events directly reduce the probability of self-fertilization. Therefore, heterogeneity in pollinator visitation among plants can produce a positive correlation of selfing.

We also found a high multilocus correlation in outcrossed paternal parentage $\left(r_{p}\right.$, Table 2$)$ in the high herkogamy group, suggesting that each plant is typically pollinated by a limited number of male parents. In insect-pollinated plants, the effective number of mates is primarily determined by pollinator movements. When the density of flowering plants is low, pollinators may move from flower to flower within a single plant (Karron et al, 1995), limiting the pollen movement among plants, such that most carry-over outcross pollen comes from neighboring plants. Variation in plant size (or number of open flowers) also reduces the effective number of mates, because large individuals may dominate the pollen pool. Similarly, many other factors, such as sexual selection through male-male competition or female mate choice (Willson and Burley, 1983), can cause correlations in outcrossed paternal parentage. Although we do not know the cause of the high correlation in our study, high correlations in outcrossed paternal parentage appear to be the rule rather than an exception in insect-pollinated plants (Morgan and Barrett, 1990). 


\section{Temporal variation in outcrossing rates}

Our population-level outcrossing-rate estimate (0.444). is somewhat lower than an estimate from the same population, 15 years prior to our study (0.75; Schoen, 1982a). One caveat is that the plants used for outcrossingrate analysis were not randomly sampled from the population; that is, we sampled plants with high and low herkogamy. The difference between our estimates and that of Schoen (1982a) may be due to temporal variation in some environmental factors such as pollinator availability. A few studies have investigated temporal variation in outcrossing rates between consecutive years; some studies reported relatively consistent outcrossing rates across consequent years (Humphreys and Gale, 1974; Kesseli and Jain, 1985; Holtsford and Ellstrand, 1989; El-Kassaby et al, 1993), whereas others reported large temporal variation similar to what we found in this study (Moran and Brown, 1980; Barrett et al, 1993; Kalisz and Vogler, 2003). However, we cannot rule out a possible, but unlikely explanation that sampling bias in our study caused the underestimation of the outcrossing rate. The plants we used may have been slightly larger than the population average because we could not collect enough fruits for our purpose from small plants. In some other species, large plants with many flowers (or a larger floral display) have lower outcrossing rates than small plants because of the increased opportunity for among-flower self-fertilization (geitonogamy; Klinkhamer and de Jong, 1993; Snow et al, 1996; Vrieling et al, 1999).

Large temporal fluctuations in outcrossing will have several implications for theories of mating-system evolution. If inbreeding depression is caused by a slow build-up of deleterious mutations, an occasional reduction in outcrossing may rapidly purge inbreeding depression and increase the opportunity for selfers to invade. Considering such effects of temporal fluctuation, Barrett et al (1993) suggested that the harmonic mean of $t$ across several generations may be biologically more relevant in characterizing the mating system than the arithmetic mean of $t$ over generations. This suggests that outcrossing rates from a single generation may underestimate the inbreeding history of the populations and the accuracy of predictions of equilibrium inbreeding depression may be limited with a point estimate of outcrossing rate.

Additionally, temporal variation in pollinator availability will further emphasize the importance of the ecological advantage of selfing-reproductive assurance (Sakai and Ishii, 1999). Since complete outcrossers will have no fitness after a season without pollinator service, occasional reduction in pollinators may be enough to maintain the residual selfing habits often observed in highly outcrossing species (Stephenson et al, 2000).

\section{Effects of herkogamy on outcrossing rate}

We observed a strong, positive association between levels of herkogamy and outcrossing rates. The results support the role of anther-stigma separation in decreasing individual selfing rate, as reported in other diverse taxa such as Lycopersicon pimpinellifolium (Rick et al, 1978), Datura stramonium (Motten and Stone, 2000), and Aquilegia caerulea (Brunet and Eckert, 1998).
Although herkogamy has traditionally been interpreted as a mechanism to restrict deleterious consequences of self-fertilization, many dichogamous or herkogamous species are equipped with additional mechanisms to promote outcrossing, such as selfincompatibility. This observation encouraged the proposal of alternative roles for herkogamy (Webb and Lloyd, 1986; Barrett, 2003). In such self-incompatible plants, herkogamy may have evolved solely to prevent the interference between the two functions of a perfect flower: pollen export from anthers (male function) and pollen import to the stigma (female function). For example, the stigma may interfere with pollen export if pollen from the anther lands on the stigma instead of on the pollinator owing to close proximity of anther and stigma (Fetscher, 2001). Likewise, anthers may interfere with pollen importation if self pollen clogs the stigma (Galen et al, 1989; Waser and Price, 1991), or if the anthers tightly surround the stigma and physically block pollinator access to the stigma. In self-compatible plants, the role of herkogamy in reducing functional interference is confounded with its role in inbreeding depression avoidance. This is because the reduction of selfing should indeed improve the efficiency of pollen exportation and reduce the possibility of stigma clogging. Therefore, it is not easy to determine whether herkogamy has evolved and been maintained due to avoidance of inbreeding depression or avoidance of interference between the sexual functions. We have shown that, in G. achilleifolia, herkogamy is correlated with a reduction in self-fertilization. Future work to determine if the degree of herkogamy also influences the effectiveness of pollen export will be valuable.

The relatively low population-level outcrossing rate in our study may have facilitated the detection of the association between herkogamy and outcrossing rate. G. achilleifolia is a protandrous, delayed selfer. The three stigma lobes are closed and not receptive when the anthers dehisce (male phase). After 26-32 h, the lobes start to curl back (hermaphrodite phase) and gradually reach a fully curled position after $2-4$ days. The stigmas of flowers with high anther-stigma distance do not touch the anthers even in the fully curled position, and the only way for selfing to occur is through pollinator visitation (facilitated autogamy). In contrast, flowers with a short anther-stigma distance have a shorter window of time available for outcrossing, because autonomous selfing (self-fertilization without the aid of pollinators) can occur shortly after the stigma starts to curl. Therefore, if pollinators had been extremely abundant, variation in herkogamy may not have influenced the outcrossing rates as much because most or all seeds may be outcrossed before any selfing takes place. Thus, in a delayed-selfing species where pollinator abundance fluctuates from year to year, a correlation between herkogamy and outcrossing rate may not be observable every year. However, under the conditions in 1995, herkogamy clearly did influence outcrossing rates.

Although our results indicate a strong effect of herkogamy on individual outcrossing rates, a previous comparison of floral traits among seven G. achilleifolia populations failed to detect a relationship between herkogamy and outcrossing rate (Schoen, 1982a). Instead, population-level outcrossing rates were positively correlated with the degree of protandry and negatively 
correlated with autonomous fruit set (Schoen, 1982a). The difference between the two studies suggests that the factors controlling variation in outcrossing rate within populations may not be always identifiable in inter-populational studies. Ecological differences among populations, such as differences in plant density or pollinator fauna, can influence the outcrossing rates (Becker et al, 1992). Such populational differences may obscure the relationship between floral traits and outcrossing, whereas plants within a single population generally experience similar pollinators (Rick et al, 1978).

Several environmental factors and other floral traits are known to influence individual outcrossing rates, as discussed in the introduction. As our goal was to assess the degree to which naturally occurring variation in herkogamy influences the outcrossing rate, other factors were not controlled at all. The results of this investigation do not preclude the possibility that other untested factors might influence the extent of outcrossing. Additionally, since our results are correlational, we cannot rule out the possibility that herkogamy is genetically or environmentally correlated with other traits, therefore influencing the outcrossing rate indirectly. Nonetheless, our results show that herkogamy can be a good indicator of individual outcrossing rate at least within this population of G. achilleifolia.

\section{Mating-system evolution}

Within-population variation in outcrossing rates has the potential to influence the coevolution between inbreeding depression and mating system through lineage specific purging of inbreeding depression (Uyenoyama and Waller, 1991). A previous study on the same G. achilleifolia population studied herein showed an association between levels of inbreeding depression and herkogamy (Takebayashi and Delph, 2000), which may be the outcome of such purging. In order to understand the cause of this correlation, it is necessary to examine the system in more detail, from multiple angles. One hypothesis to explain the correlation is that it was caused by differences in inbreeding history among lineages. There are several assumptions which need to be tested to determine if this hypothesis is plausible. In this paper, we have addressed one part of the unsolved puzzle. Our results show that herkogamy can influence the outcrossing rate in the wild, and the effect appears to be moderately large. This gives plausibility to the hypothesis that the correlation in Takebayashi and Delph (2000) could be the result of lineage-dependent purging. The other assumption which we need to test is whether herkogamy is heritable in this species. After we gather data about all parts of the puzzle, we will be able to address the implication of correlation between mating system modifiers and inbreeding depression.

\section{Acknowledgements}

We thank MF Dybdahl, GJ Gastony, M Jensen, and DL Marr for help with isozyme electrophoresis; DE Krause and PL Morrell for help with the field work; CM Lively and LH Rieseberg for helpful comments on the manuscript. This work was supported by grants from the Department of Biology at Indiana University, the Indiana Academy of Science, Sigma Xi, Hardman Native Plant Research Award from California Native Plant Society, the
National Science Foundation, NSF Alaska EPSCoR (EPS0346770), and NIH Alaska INBRE (2P20RR16466).

\section{References}

Baker HG (1955). Self-compatibility and establishment after 'long distance' dispersal. Evolution 9: 347-349.

Barrett SCH (2003). Mating strategies in flowering plants: the outcrossing-selfing paradigm and beyond. Phil Trans $R$ Soc London, Series B, Biological Sciences 358: 991-1004.

Barrett SCH, Eckert CG (1990). Variation and evolution of mating systems in seed plants. In: Kawano S (ed) Biological Approaches and Evolutionary Trends in Plants. Academic Press: New York, NY, USA. pp 229-254.

Barrett SCH, Harder LD, Cole WW (1994). Effects of flower number and position on self-fertilization in experimental populations of Eichhornia paniculata (Pontederiaceae). Functional Ecology 8: 526-535.

Barrett SCH, Husband BC, Cole WW (1993). Variation in outcrossing rates in Eichhornia paniculata: Temporal changes in populations of contrasting style morph structure. Plant Species Biology 8: 141-148.

Becker HC, Damgaard C, Karlsson B (1992). Environmental variation for outcrossing rate in rapeseed (Brassica napus). Theor Appl Genet 84: 303-306.

Belaoussoff S, Shore JS (1995). Floral correlates and fitness consequences of mating-system variation in Turnera ulmifolia. Evolution 49: 545-556.

Brown AHD, Allard RW (1970). Estimates of the mating systems in open-pollinated maize populations using isozyme polymorphisms. Genetics 66: 133-145.

Brunet J, Eckert CG (1998). Effects of floral morphology and display on outcrossing in blue columbine, Aquilegia caerulea (Ranunculaceae). Functional Ecology 12: 596-606.

Carr DE, Fenster CB, Dudash MR (1997). The relationship between mating-system characters and inbreeding depression in Mimulus guttatus. Evolution 51: 363-372.

Cruzan MB, Hamrick JL, Arnold ML, Bennett BD (1994). Mating-system variation in hybridizing irises: effects of phenology and floral densities on family outcrossing rates. Heredity 72: 95-105.

Darwin CR (1876). The Effect of Cross and Self Fertilization in the Vegetable Kingdom. John Murray: London.

El-Kassaby YA, Meagher MD, Davidson R (1993). Temporal variation in the outcrossing rate in a natural stand of Wesetern white-pine. Silvae Genetica 42: 131-135.

Fetscher AE (2001). Resolution of male-female conflict in an hermaphroditic flower. Proc $R$ Soc London Series $B$ 268: 525-529.

Galen C, Gregory T, Galloway LF (1989). Costs of selfpollination in a self-incompatible plant, Polemonium viscosum. Am J Botany 76: 1675-1680.

Grant V, Grant KA (1965). Flower Pollination in the Phlox Family. Columbia University Press: New York, NY, USA.

Hebert PDN, Beaton MJ (1989). Methodologies for Allozyme Analysis Using Cellulose Acetate Electrophoresis. Helena Laboratories: Beaumont, Texas.

Holtsford TP, Ellstrand NC (1989). Variation in outcrossing rate and population genetic structure of Clarkia tembloriensis (Onagraceae). Theor Appl Genet 78: 480-488.

Holtsford TP, Ellstrand NC (1992). Genetic and environmental variation in floral traits affecting outcrossing rate in Clarkia tembloriensis (Onagraceae). Evolution 46: 216-225.

Humphreys MO, Gale JS (1974). Variation in wild populations of Papaver dubium. VIII. The mating system. Heredity 33: 33-41.

Ivey CT, Wyatt R (1999). Family outcrossing rates and neighborhood floral density in natural populations of swamp milkweed (Asclepias incarnata): potential statistical artifacts. Theor Appl Genet 98: 1063-1071. 
Kalisz S, Vogler DW (2003). Benefits of autonomous selfing under unpredictable pollinator environments. Ecology 84: 2928-2942.

Karron JD, Thumser NN, Tucker R, Hessenauer AJ (1995). The influence of population density on outcrossing rates in Mimulus ringens. Heredity 75: 175-180.

Kesseli RW, Jain SK (1985). Breeding systems and population structure in Limnanthes. Theor Appl Genet 71: 292-299.

Kimura M (1959). Conflict between self-fertilization and outbreeding in plants. Annual Report National Institute of Genetics Japan 9: 87-88.

Klinkhamer PGL, de Jong TJ (1993). Attractiveness to pollinators: a plant's dilemma. Oikos 66: 180-184.

Kreitman M, Hudson RR (1991). Inferring the evolutionary histories of the Adh and Adh-dup loci in Drosophila melanogaster from patterns of polymorphism and divergence. Genetics 127: 565-582.

Kunin WE (1993). Sex and the single mustard: population density and pollinator behavior effects on seed-set. Ecology 74: 2145-2160.

Lande R, Schemske DW (1985). The evolution of self-fertilization and inbreeding depression in plants. I. Genetic models. Evolution 39: 24-40.

Lloyd DG, Webb CJ (1986). The avoidance of interference between the presentation of pollen and stigmas in angiosperms. I. Dichogamy. New Zealand J Botany 24: 135-162.

Loveless MD, Hamrick JL (1984). Ecological determinants of genetic structure in plant populations. Annual Rev Ecology and Systematics 15: 65-95.

Miyashita NT (2001). DNA variation in the $5^{\prime}$ upstream region of the Adh locus of the wild plants Arabidopsis thaliana and Arabis gemmifera. Mol Biol Evolution 18: 164-171.

Moran GF, Brown AHD (1980). Temporal heterogeneity of outcrossing rate in alpine ash (Eucalyptus delegatensis RT Bak). Theor Appl Genet 57: 101-105.

Morgan MT, Barrett SCH (1990). Outcrossing rates and correlated mating within a population of Eichhornia paniculata (Pontederiaceae). Heredity 64: 271-280.

Motten AF, Stone JL (2000). Heritability of stigma position and the effect of stigma-anther separation on outcrossing in a predominantly self-fertilizing weed, Datura stramonium (Solanaceae). Am I Botany 87: 339-347.

Mutikainen P, Delph LF (1998). Inbreeding depression in a gynodioecious Lobelia siphilitica: among-family differences override between-morph differences. Evolution 52: 1572-1582.

Rao GY, Widen B, Andersson S (2002). Patterns of inbreeding depression in a population of Brassica cretica (brassicaceae): Evidence from family-level analyses. Biological J Linnean Soc 76: $317-325$.

Richardson BJ, Baverstock PR, Adams M (1986). Allozyme Electrophoresis: A Handbook for Animal Systematics and Population Studies. Academic Press: Sydney.

Rick CM, Fobes JF, Holle M (1977). Genetic variation in Lycopersicon pimpinellifolium: evidence of evolutionary change in mating systems. Plant Systematics and Evolution 127: 139-170.

Rick CM, Holle M, Thorp RW (1978). Rates of cross-pollination in Lycopersicon pimpinellifolium: Impact of genetic variation in floral characters. Plant Systematics and Evolution 129: 31-44.

Ritland K (1988). The genetic-mating structure of subdivided populations. II. Correlated mating models. Theor Population Biol 34: 320-346.

Ritland K (2002). Extensions of models for the estimation of mating systems using $n$ independent loci. Heredity 88: 221228.

Ritland K, Ganders FR (1985). Variation in the mating system of Bidens menziesii (Asteraceae) in relation to population substructure. Heredity 55: 235-244.
Ritland K, Jain S (1981). A model for the estimation of outcrossing rate and gene frequencies using $n$ independent loci. Heredity 47: 35-52.

Sakai S, Ishii HS (1999). Why be completely outcrossing? Evolutionarily stable outcrossing strategies in an environment where outcross-pollen availability is unpredictable. Evolutionary Ecology Res 1: 211-222.

Savolainen O, Langley CH, Lazzaro B, Freville H (2000). Contrasting patterns of nucleotide polymorphism at the alcohol dehydrogenase locus in the outcrossing Arabidopsis lyrata and the selfing Arabidopsis thaliana. Mol Biol Evol 17: 645-655.

Schoen DJ (1982a). The breeding system of Gilia achilleifolia: variation in floral characteristics and outcrossing rate. Evolution 36: 352-360.

Schoen DJ (1982b). Genetic variation and the breeding system of Gilia achilleifolia. Evolution 36: 361-370.

Schultz ST, Willis JH (1995). Individual variation in inbreeding depression: the roles of inbreeding history and mutation. Genetics 141: 1209-1223.

Snow AA, Spica TP, Simpson R, Klips RA (1996). The ecology of geitonogamous pollination. In: Lloyd DG, Barrett SCH (eds) Floral Biology: Studies on Floral Evolution in Animal-Pollinated Plants. Chapman and Hall: New York. pp 191-216.

Stephenson AG, Good SV, Vogler DW (2000). Interrelationships among inbreeding depression, plasticity in the self-incompatibility system, and the breeding system of Campanula rapunculoides L. (Campanulaceae). Ann Botany 85: 211-219.

Stone JL, Motten AF (2002). Anther-stigma separation is associated with inbreeding depression in Datura stramonium, a perdominantly self-fertilizing annual. Evolution 56: 21872195.

Takebayashi N, Delph LF (2000). An association between a floral trait and inbreeding depression. Evolution 54: 840-846.

Takebayashi N, Morrell PL (2001). Is self-fertilization an evolutionary dead end? revisiting an old hypothesis with genetic theories and a macroevolutionary approach. Am J Botany 88: 1143-1150.

Ushimaru A, Nakata K (2002). The evolution of flower allometry in selfing species. Evolutionary Ecology Res 4: $1217-1227$.

Uyenoyama MK, Waller DM (1991). Coevolution of selffertilization and inbreeding depression. I. Mutation-selection balance at one and two loci. Theoretical Population Biol 40: $14-46$.

Vogler DW, Filmore K, Stephenson AG (1999). Inbreeding depression in Campanula rapunculoides L. I. A comparison of inbreeding depression in plants derived from strong and weak self-incompatibility phenotypes. J Evolutionary Biol 12: 483-494.

Vrieling K, Saumitou-Laprade P, Cuguen J, van Dijk H, de Jong TJ, Klinkhamer PGL (1999). Direct and indirect estimates of the selfing rate in small and large individuals of the bumblebee pollinated Cynoglossum officinale L (Boraginaceae). Ecology Lett 2: 331-337.

Waser NM, Price MV (1991). Reproductive costs of selfpollination in Ipomopsis aggregata (Polemoniaceae). Am J Botany 78: 1036-1043.

Webb CJ, Lloyd DG (1986). The avoidance of interference between the presentation of pollen and stigmas in angiosperms. II. Herkogamy. New Zealand I Botany 24: 163-178.

Willson MF, Burley N (1983). Mate Choice in Plants: Tactics, Mechanisms, and Consequences. Princeton University Press: Princeton, NJ.

Wright S, Lauga B, Charlesworth D (2002). Rates and patterns of molecular evolution in inbred and outbred Arabidopsis. Mol Biol Evol 19: 1407-1420. 